\title{
Deficiency of platelet-activating factor acetylhydrolase is a severity factor for asthma
}

\author{
Diana M. Stafforini, ${ }^{1}$ Toshio Numao, ${ }^{2}$ Alexander Tsodikov, ${ }^{1}$ Darius Vaitkus, ${ }^{1}$ \\ Takeshi Fukuda, ${ }^{3}$ Naoto Watanabe, ${ }^{3}$ Naoto Fueki, ${ }^{3}$ Thomas M. McIntyre, ${ }^{4}$ \\ Guy A. Zimmerman, ${ }^{4}$ Sohei Makino, ${ }^{2}$ and Stephen M. Prescott ${ }^{1}$
}

${ }^{1}$ Huntsman Cancer Institute, Program in Human Molecular Biology and Genetics, Eccles Institute of Human Genetics,
University of Utah, Salt Lake City, Utah 84112-5332, USA
2Department of Medicine and Clinical Immunology, Dokkyo University School of Medicine, Tochigi, 321-0207, Mibu, Japan
${ }^{3}$ Department of Medicine, Jobu NK Hospital, Maebasahi, 371-0048, Gunma, Japan
${ }^{4}$ Cardiovascular Research and Training Institute, University of Utah, Salt Lake City, Utah 84112-5332, USA

Address correspondence to: Diana M. Stafforini, Program in Human Molecular Biology and Genetics,

University of Utah, 15 North 2030 East, Room 2100, Salt Lake City, Utah 84112-5332, USA. Phone: (801) 585-3402;

Fax: (801) 585-6345; E-mail: diana.stafforini@hci.utah.edu

Received for publication October 21, 1998, and accepted in revised form February 17, 1999.

\begin{abstract}
Asthma, a family of airway disorders characterized by airway inflammation, has an increasing incidence worldwide. Platelet-activating factor (PAF) may play a role in the pathophysiology of asthma. Its proinflammatory actions are antagonized by PAF acetylhydrolase. A missense mutation (V279F) in the PAF acetylhydrolase gene results in the complete loss of activity, which occurs in $4 \%$ of the Japanese population. We asked if PAF acetylhydrolase deficiency correlates with the incidence and severity of asthma in Japan. We found that the prevalence of PAF acetylhydrolase deficiency is higher in Japanese asthmatics than healthy subjects and that the severity of this syndrome is highest in homozygous-deficient subjects. We conclude that the PAF acetylhydrolase gene is a modulating locus for the severity of asthma.
\end{abstract}

J. Clin. Invest. 103:989-997

\section{Introduction}

The marked increase in the prevalence of asthma has made it a global health concern (1). Our understanding of the basic mechanisms of asthma has increased significantly, but the heterogeneous nature of clinical manifestations and therapeutic responses in both adult and pediatric patients has prevented a breakthrough discovery (2). The perception of asthma has shifted from a disease primarily characterized by altered smooth muscle function to one mainly characterized by acute, subacute, and/or chronic inflammation driven by a variety of agents $(2,3)$. The patterns of pathologic changes in the bronchioles and bronchi and the degree of reversibility vary among individual patients and patient subsets. Because it is now widely accepted that asthma, in its varied forms, is an inflammatory disorder of the airways in which mediators released from activated mast cells and eosinophils play a major role $(1,3)$, many recent strategies for new treatments have focused on control of the underlying inflammation by matching each mediator with a specific inhibitor (2). Consequently, there has been caution regarding the overuse of corticosteroids, which are potent nonspecific anti-inflammatory drugs that may have adverse effects when used for long periods, either systematically or by inhalation in high doses.

Inflammation is a complex process that involves numerous types of cells and the molecules that activate them; these networks of cells and signaling molecules interact to create redundant pathways (4). There likely are several levels at which molecular intervention may attenuate inflammatory responses and ameliorate symp- toms. Platelet-activating factor (PAF, 1-O-alkyl-2-acetylsn-glycero-3-phosphocholine) has been implicated in the pathophysiology of inflammation in asthma. PAF is a chemotactic lipid that activates inflammatory cells, including eosinophils, and causes microvascular leakage, bronchoconstriction, and airway hyperresponsiveness. In addition, PAF causes the release of other inflammatory agonists from eosinophils, and eosinophils from asthmatics exhibit a markedly increased sensitivity to PAF compared with those from normal (nonasthmatic) subjects (5). PAF is produced by a variety of cell types involved in asthma, including alveolar macrophages, platelets, and eosinophils (6). However, the precise role that PAF plays in the pathophysiology of asthma is controversial, and multiple approaches have been used to examine its contribution (7). For example, blood PAF levels of asthmatic subjects with active symptoms were found to be significantly higher than those of patients during remission or healthy volunteers, suggesting that PAF may play a role in bronchial asthma $(8,9)$. Increased levels of PAF also have been reported in children with acute asthmatic attacks (10). The desensitized state of human platelets has been used as an index of previous exposure to PAF and was shown to correlate with responsiveness to inhalation of specific allergens (11). Transgenic mice expressing the PAF receptor are more susceptible to methacholine-induced bronchial hypersensitivity than normal littermates (12), and a significant increase in PAF receptor mRNA levels has been reported in the lungs of asthmatic patients (13). Finally, PAF receptor antagonists have also been used to indirectly examine 
Table1

Selected characteristics of normal and asthmatic subjects

\begin{tabular}{lcccc}
\hline \multirow{2}{*}{ Parameter analyzed } & \multicolumn{2}{c}{ Men } & Women \\
& Control & Asthmatic & Control & Asthmatic \\
Number of subjects & $153(58.2 \%)$ & $119(44.7 \%)$ & $110(41.8 \%)$ & $147(55.3 \%)$ \\
Average years (range) & $43.7(21-80)$ & $48.7(11-78)$ & $43.9(18-90)$ & $48.6(15-77)$ \\
Positive smoking status & $85(55.6 \%)$ & $18(15.1 \%)$ & $18(16.4 \%)$ & $11(7.5 \%)$ \\
\hline
\end{tabular}

constitutes a risk factor for the development of asthma, suggesting that the PAF acetylhydrolase locus may modulate the severity of this syndrome in Japanese adults. This observation in a select population is relevant to a larger population as PAF acetylhydrolase activity levels vary widely.

\section{Methods}

Patients. We studied 264 asthmatic subjects and 263 age-matched, healthy subjects from participating hospitals of the Tochigi, Gunma, and

the involvement of PAF in asthma. The initial clinical trials of such compounds were negative (14), perhaps because of features of PAF and related lipids that make the problem difficult to approach by receptor blockade (15). However, recent studies with newly developed PAF receptor antagonists have been positive, which suggests that PAF (and/or structurally related analogues) is likely a key mediator (16-18). In animal studies, PAF was implicated as an intermediate in asthma because receptor antagonists blocked physiological responses in protocols in which an immunological response was provoked $(19,20)$. In summary, several independent lines of evidence indicate that PAF is involved in asthma as a mediator of positive feedback mechanisms during the inflammation that occurs in asthmatic airways (6).

An alternative to treating asthma by using competitive receptor blockade to antagonize the proinflammatory actions of PAF and terminate its signaling is PAF degradation (21), which occurs by removal of its 2 -acetyl group. This reaction is catalyzed by PAF acetylhydrolases, a family of enzymes that exist in cytosolic and secreted forms (22). A decrease in PAF acetylhydrolase activity would be predicted to exaggerate inflammatory and allergic responses involving PAF. Miwa et al. previously reported that $4 \%$ of the Japanese population is deficient in PAF acetylhydrolase activity in plasma, and that the prevalence of deficiency of the enzyme is higher in children with severe asthma (23). We recently found that a point mutation (V279F) near the active site of PAF acetylhydrolase results in the replacement of valine 279 with phenylalanine, and that this mutation results in an inactive enzyme (24). About one-third of the Japanese population is heterozygous for this mutation.

A genetic abnormality that leads to impaired hydrolysis of PAF has important implications for asthma because there is a strong inheritable component to this syndrome $(25,26)$. For example, naturally occurring mutations in the human 5-lipoxygenase gene promoter have been observed in asthmatic patients, but not in normal subjects (27). However, the inheritance pattern of asthma is complex and cannot be classified by traditional models; the heterogeneity of the disease complicates the issue further. In the studies reported here we asked if the prevalence of PAF acetylhydrolase deficiency is higher in Japanese asthmatics compared with an age-matched group of subjects without asthma, and if the level of PAF acetylhydrolase activity in plasma correlates with the severity of asthma. We report that a partial or complete deficiency in PAF acetylhydrolase activity
Tokyo prefectures in eastern Japan. We also analyzed 179 samples from a white North American population. Informed consent was obtained from all subjects or their guardians. Blood was drawn by venipuncture, using sodium citrate as an anticoagulant. Plasma was isolated by centrifugation at 2,500 $\mathrm{g}$ for $10 \mathrm{~min}$ at $4{ }^{\circ} \mathrm{C}$. The remaining blood cells were saved for genomic DNA isolation (see below). The patients were selected according to national and international standards for the diagnosis of asthma (28). Information regarding smoking status, age, and past history of asthmatic attacks was obtained from each patient. We categorized the subjects based on their symptoms and peak flow monitoring into four severity groups (mild intermittent, mild persistent, moderate persistent, and severe ). In addition, we created four categories based on the level of steroids used (none, $0-5$ $\mathrm{mg} /$ day, $5-10 \mathrm{mg} /$ day, and > $10 \mathrm{mg} /$ day). Finally, we recorded whether the patients were in remission or undergoing an attack at the time of their visit to the clinic.

PAF acetylhydrolase assays. PAF (1-O-alkyl-2-acetyl-sn-glycero3-phosphocholine) was purchased from Avanti Polar Lipids Inc., Alabaster, Alabama, USA. PAF acetylhydrolase activity was determined by incubation with [ ${ }^{3} \mathrm{H}$-acetyl]PAF (Du Pont NEN Research Products, Boston, Massachusetts, USA) and separation of the reaction products by reverse-phase liquid chromatography as described previously (29).

Amplification of genomic DNA. Genomic DNA was isolated from blood cells using a commercially available genomic DNA isolation kit (Amersham Life Sciences Inc., Arlington Heights, Illinois, USA). The genotype at position 279 of the PAF acetylhydrolase gene was determined by a PCR-based, allele-specific method previously described (24).

Site-directed mutagenesis. Constructs harboring the Q281R and I198T mutations were obtained by oligonucleotide-directed mutagenesis of these sites as described elsewhere (30). The presence of the desired mutations was established by automated DNA sequence analysis. The mutants were expressed in a pUC vector to which the tryptophan promoter was added to allow expression of PAF acetylhydrolase in E. coli (30). The promoter was derepressed by depletion of tryptophan, which occurs during overnight incubation at $37^{\circ} \mathrm{C}$. The cultures were harvested by centrifugation, and the cells were resuspended in buffer $\mathrm{A}$, consisting of $100 \mathrm{mM}$ succinate, $100 \mathrm{mM} \mathrm{NaCl}, 1$

\section{Table 2}

Genotype distribution at position 279 of PAF acetylhydrolase in Japanese asthmatics and healthy controls

$\begin{array}{lccc}\text { Genotype at position } 279 & \text { Category } & \text { Number of subjects } & \text { Percentage } \\ \text { Normal } & \text { Normal } & 177 & 67.3 \\ & \text { Asthmatic } & 158 & 59.4 \\ \text { Heterozygous } & \text { Normal } & 78 & 29.7 \\ & \text { Asthmatic } & 96 & 36.1 \\ \text { Homozygous } & \text { Normal } & 8 & 3.0 \\ & \text { Asthmatic } & 12 & 4.5\end{array}$




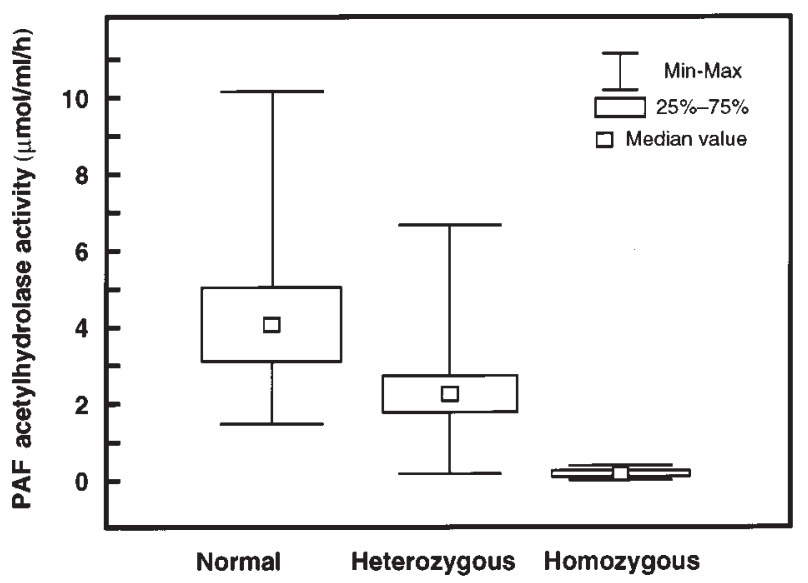

Figure 1

Distribution of PAF acetylhydrolase activity according to genotype. Plasma samples from control subjects and from asthmatic patients were assayed for PAF acetylhydrolase activity as described previously (29). The genotype at the 279 locus was determined by an allele-specific PCR assay (24), and the subjects were categorized according to genotype. The values reported are the average of two determinations that agreed within $10 \%$ of each other.

mM EDTA, and 20 mM CHAPS (pH 6.0). Cells were then lysed by sonication and allowed to incubate for $60 \mathrm{~min}$ on ice. The soluble fraction was recovered by centrifugation and the pellet was discarded.

Immunoblots. Supernatants were assayed for PAF acetylhydrolase activity and subjected to immunoblot analysis as described (30). The blots were developed using a polyclonal anti-PAF acetylhydrolase antiserum. The antiserum was prepared by immunization of New Zealand white rabbits with the purified recombinant protein. The primary antibody was used at a 1:2,000 dilution. A horseradish peroxidase-labeled goat anti-rabbit IgG antibody (diluted 1:5,000; purchased from Kirkegaard \& Perry Laboratories Inc., Gaithersburg, Maryland, USA), was used as the secondary antibody. The blots were developed using the enhanced chemiluminescent substrate kit from Amersham Life Sciences, Inc.

Statistical analysis. In a first attempt to relate asthma severity to various explanatory variables, we regressed the presence of asthma attacks (a binary response variable) on PAF acetylhydrolase activity, genotype (normal, heterozygous for the V279F mutation, and homozygous for V279F), age, sex, smoking status (nonsmoking or smoking), type of asthma (atopic or nonatopic), and use of steroids (none, $0-5 \mathrm{mg} /$ day, 5-10 $\mathrm{mg} /$ day, and $>10 \mathrm{mg} /$ day). It should be noted that the use-ofsteroids factor has a dual character, steroids being anti-asthma drugs and surrogate response markers at the same time. Indeed, the progress of asthma is associated with an increased administration of the drug. For this reason the use of steroids would still be expected to be the strongest "predictor" of asthma severity if included in the model, even if steroids had no effect on the course of the disease. Because of the difficulty in separating these opposite effects, we performed two analyses in which steroids were either included or excluded from the model. The probability of having no asthma attacks in the past was related in a logistic regression model to a linear predictor based on the above-mentioned explanatory variables.

To review the clinical information regarding the severity of asthma at the time the samples were collected (ranked as mild intermittent, mild persistent, moderate persistent, and severe, we used the proportional Odds regression test $(31,32)$ which provides a more sensible average over the three divergent analyses.

\section{Results}

PAF acetylhydrolase activity in plasma from Japanese asthmatic patients and normal controls. In this study we analyzed results from 264 asthmatics and 263 healthy subjects with normal gender distribution from eastern Japan (Tochigi, Gunma and Tokyo prefectures). Age, sex, and smoking status of the two groups are summarized in Table 1 . The average age was similar in the two groups, with a slightly higher average age in the asthmatic group (both for men and women). There was a much higher proportion of normal males who smoked (55.6\%) than normal females (16.4\%). We determined PAF acetylhydrolase activity in the plasma derived from these samples and compared the average activity in the two groups. We found that the mean activities in samples from asthmatics were lower than in samples from the control groups $(3.08 \pm 1.52$ and $3.56 \pm 1.67 \mu \mathrm{mol} / \mathrm{ml} /$ hour, respectively; $P<0.0001)$, which agrees with previous studies $(33,34)$. With a rather large sample size the $t$-test for comparison of the mean PAF acetylhydrolase activities in asthmatics and normals has a high power (92\%). For this reason a relatively small difference turns out to be highly significant. Although this difference could have been detected with a smaller sample size of 180 per group with a power of at least $80 \%$, a larger study was designed to achieve better sensitivity for regression analyses of asthma severity scores and variable selection procedures.

We found a strong association between PAF acetylhydrolase activity and genotype using asthmatic and normal samples combined (Figure 1). The variance test analysis rejected the equality of means among the three categories $(P<0.01)$. The mean PAF acetylhydrolase activity decreased steeply from normal $(4.09 \pm 1.38)$ to heterozygous $(2.22 \pm 0.77)$ and homozygous subjects $(0.05 \pm 0.10)$. The distribution of activity in the study

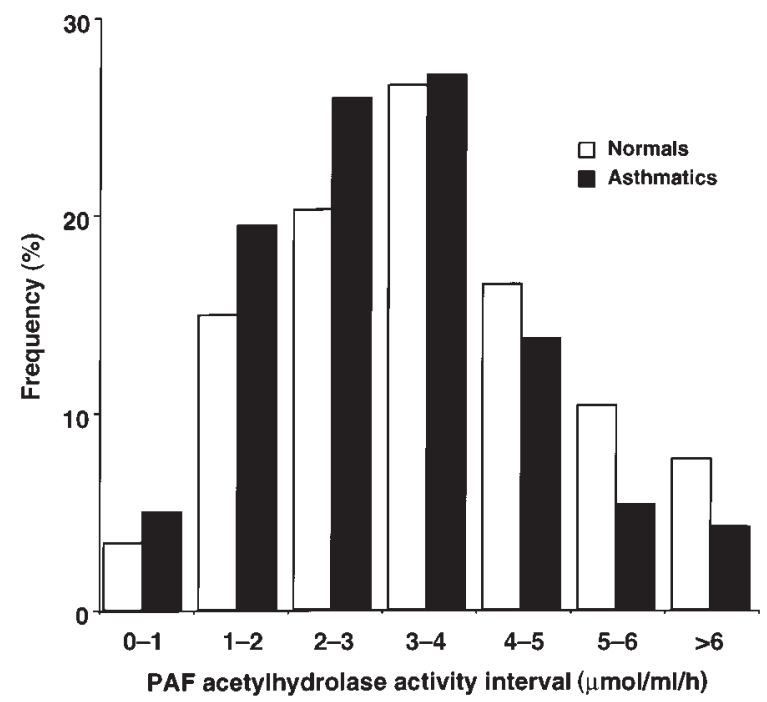

Figure 2

Distribution of PAF acetylhydrolase activity in normal and asthmatic patients. Plasma samples from control subjects and from asthmatic patients were assayed for PAF acetylhydrolase activity as described (29). The activity values were divided into seven groups of incremental activity and plotted as percentages of the total for each category. The values reported are the average of two determinations that agreed within $10 \%$ of each other. 


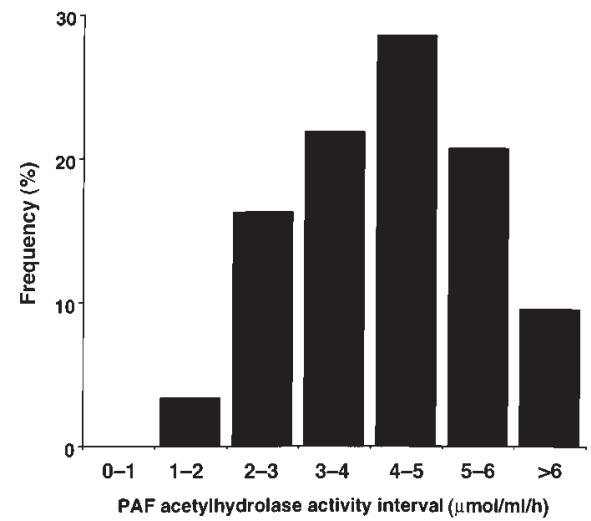

Figure 4

Distribution of PAF acetylhydrolase activity in white subjects. The plasma PAF acetylhydrolase activity values from white subjects were divided into seven groups of incremental activity and plotted as percentages of the total for each category. The values reported are the average of two determinations that agreed within $10 \%$ of each other.

group was virtually identical to what we previously observed in subjects from a different region of Japan (24). The frequency of asthmatics with PAF acetylhydrolase activity below the normal mean of $3.5 \mathrm{U} / \mathrm{ml}$ was higher than the corresponding frequency in the control group (Figure 2). Also, the frequency of control subjects with PAF acetylhydrolase activity higher than the mean was greater than the frequency of asthmatics in the same PAF acetylhydrolase interval (Figure 2). Smoking results in the inhibition of PAF acetylhydrolase activity $(35,36)$, and so the comparatively high number of smokers in the control group may have caused an underrepresentation of the actual difference. These results suggest that the level of PAF acetylhydrolase activity and the clinical syndrome of asthma are related.

Previous studies have demonstrated that steroids regulate the levels of PAF acetylhydrolase activity in vivo (37, 38). We found no correlation between the total activity in plasma and the level of steroids used by asthmatic patients. Predictably, patients that used the highest steroid doses (>10 mg/day) had the most severe form of asthma and the highest incidence of attacks compared with asthmatics who used low levels of steroids (0-5 mg/day).

Relationship between PAF acetylbydrolase activity and genotype at the V279F locus. To examine if the differences in PAF acetylhydrolase distribution between the normal and asthmatic groups resulted from the mutation that we previously identified (V279F), we determined the genotype of each sample, as described (24). We found (Table 2) that the prevalence of the V279F mutation was higher in the asthmatic group than in the control, both as a homozygous and a heterozygous trait. Conversely, there were fewer subjects with two normal alleles in the asthmatic group (Table 2). We next divided the asthmatic group according to genotype, i.e., heterozygous or normal (homozygous subjects were excluded from this analysis), and examined the distribution of activity (Figure 3). We found that the mutation at position 279 accounted almost entirely for the altered distribution of activity in the asthmatic group, as segregation of the groups according to genotype showed enzymatic activities that were distributed normally, but centered at maxima that differed by a factor of two, as expected (Figure 3 ). These results also have implications for other populations, as the distribution of serum PAF acetylhydrolase activity in whites includes a significant fraction of individuals with levels lower than the mean (Figure 4).

Correlation between clinical manifestations and PAF acetylhydrolase genotype at the 279 locus. Our next goal was to determine whether, within the asthmatic group, there was a relationship between PAF acetylhydrolase deficiency and other clinical parameters. The probability of having no asthma attacks in the past was analyzed using two models that included or excluded steroid use (Table 3). Both analyses showed a significant advantageous effect of higher PAF acetylhydrolase activity on the presence of asthma attacks ( $P=0.03$ and 0.01 , respectively). This is reflected by an Odds ratio of 1.44 , which is significantly higher than 1.0 (Table 3). In contrast, age and the type of asthma had no significant effect (not shown). An interesting, yet unexplained, independent result was found when we compared patients with equal levels of PAF acetylhydrolase activity
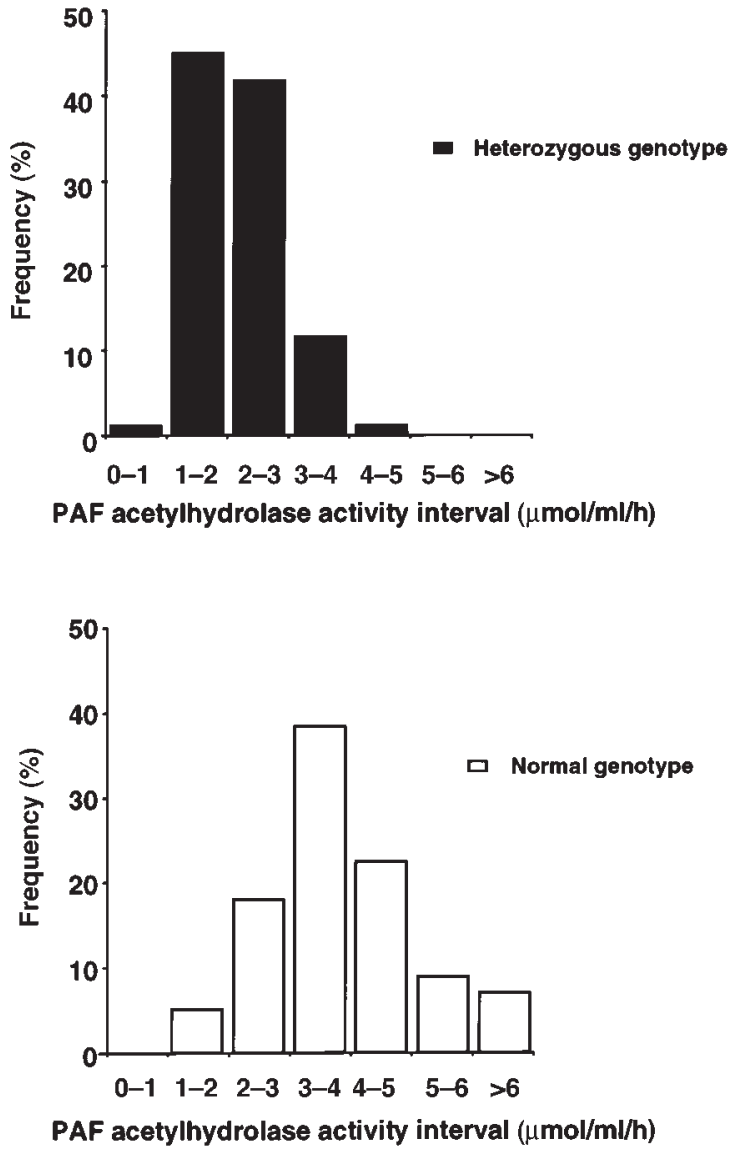

Figure 3

Distribution of PAF acetylhydrolase activity in asthmatic patients based on the genotype at position 279 . The PAF acetylhydrolase activity values from asthmatics were categorized according to the genotype at position 279. (The homozygous patients were excluded from this analysis.) The values were divided into seven groups of incremental activity and plotted as percentages of the total for each category. The values reported are the average of two determinations that agreed within $10 \%$ of each other. 


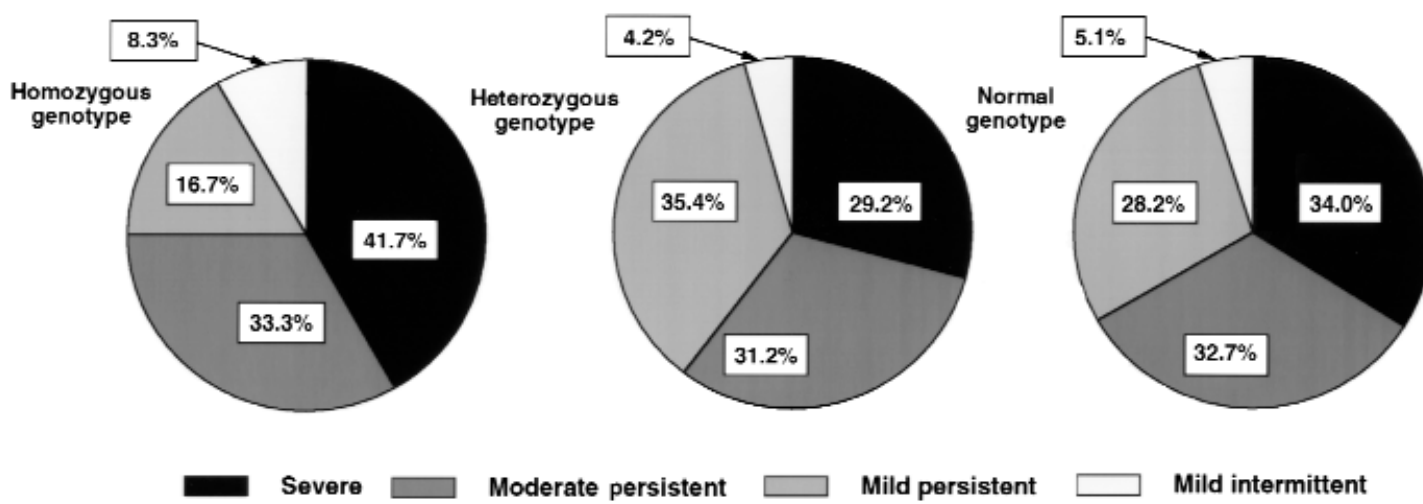

Figure 5

The severity of asthma is dependent on the genotype at position 279 . The asthmatic category was divided in four groups according to the severity of the disease. The frequency of individuals with the most severe form of asthma was highest in the homozygous group.

but different genotypes. We found that heterozygous subjects seemed to have an advantage over normals, and that homozygous subjects had an advantage over heterozygous patients in terms of the probability of having asthma attacks (Table 3)However, because genotype was a strong predictor of PAF acetylhydrolase deficiency (Figure 1), its effect was a direct one if unadjusted for PAF acetylhydrolase activity. Thus, a deficiency in PAF acetylhydrolase enzymatic activity has a detrimental effect on the incidence of asthma attacks.

We also reviewed the clinical information regarding the severity of asthma at the time the samples were collected (ranked as mild intermittent, mild persistent, moderate persistent, and severe). We found that the highest incidence of severe asthma was in patients who were homozygous for the V279F mutation (Figure 5). It is possible, however, that this apparent distribution may have been a consequence of the low total number of patients with mild intermittent asthma The presence of the V279F mutation as a heterozygous trait did not affect the severity of asthmatic symptoms compared with controls (Figure 5). In addition, there was no difference in the frequency of the V279 mutation in the patients who were undergoing an attack at the time that the blood samples were collected compared with those who were in remission. Predictably, the group of patients using high levels of steroid medication (more than $5 \mathrm{mg} /$ day) had a higher proportion of homozygous subjects (6.2\%) compared with the group that used less than $5 \mathrm{mg}$ of the medication daily (only $1.2 \%$ of the subjects in this group were homozygous for the V279F mutation). We also performed a proportional Odds Regression analysis in which the severity of asthma was characterized by a more informative response measure (see Methods). The results of the regression analysis based on the asthma severity score agreed with the logistic regression analysis of asthma attacks (Table 4). In

Table 3

\section{Table 4} of asthma the presence of steroids in the model there were trends for a significant advantageous effect of PAF acetylhydrolase activity $(P=0.12)$ and a reverse independent effect of the genotype $(P=0.07)$ dominated by a very strong effect of steroids $(P=0.0001)$. When the contribution of steroid administration was removed from the model, the effects of PAF acetylhydrolase and genotype became significant $(P=0.02$ for both), and age had a negligible effect (not shown). The proportional Odds assumption was verified by the data $(P>0.3)$.

Additional alterations in the PAF acetylhydrolase gene. We observed that one asthmatic subject had almost undetectable serum PAF acetylhydrolase activity but had an unexpected genotype. This subject, who suffered from the most severe form of asthma was expected to be homozygous for the V279F mutation, yet was found to harbor this

Regression analysis of PAF acetylhydrolase activity, genotype at the 279 locus and presence of asthma attacks

\begin{tabular}{lcccc}
\hline $\begin{array}{l}\text { Response variable } \\
\text { Presence }\end{array}$ & Steroids in the model & Factor & Odds ratio & $P$ value \\
of & & & & \\
asthma & Yes & PAF acetylhydrolase activity & 1.44 & 0.03 \\
attacks & & Genotype & 2.87 & 0.01 \\
& & Steroids & 0.43 & 0.0001 \\
& & & \\
& No & PAF acetylhydrolase activity & 1.54 & 0.01 \\
& & Genotype & 3.36 & 0.002 \\
& & & &
\end{tabular}

Regression analysis of PAF acetylhydrolase activity, genotype at the 279 locus, and severity

\begin{tabular}{|c|c|c|c|c|}
\hline Response variable & Steroids in the mod & Factor & Odds ratio & $P$ value \\
\hline Asthma & Yes & PAF acetylhydrolase activity & 1.18 & 0.12 \\
\hline Severity & & Genotype & 1.67 & 0.07 \\
\hline \multirow[t]{3}{*}{ score } & & Steroids & 0.29 & 0.0001 \\
\hline & No & PAF acetylhydrolase activity & 1.27 & 0.02 \\
\hline & & Genotype & 1.85 & 0.02 \\
\hline
\end{tabular}




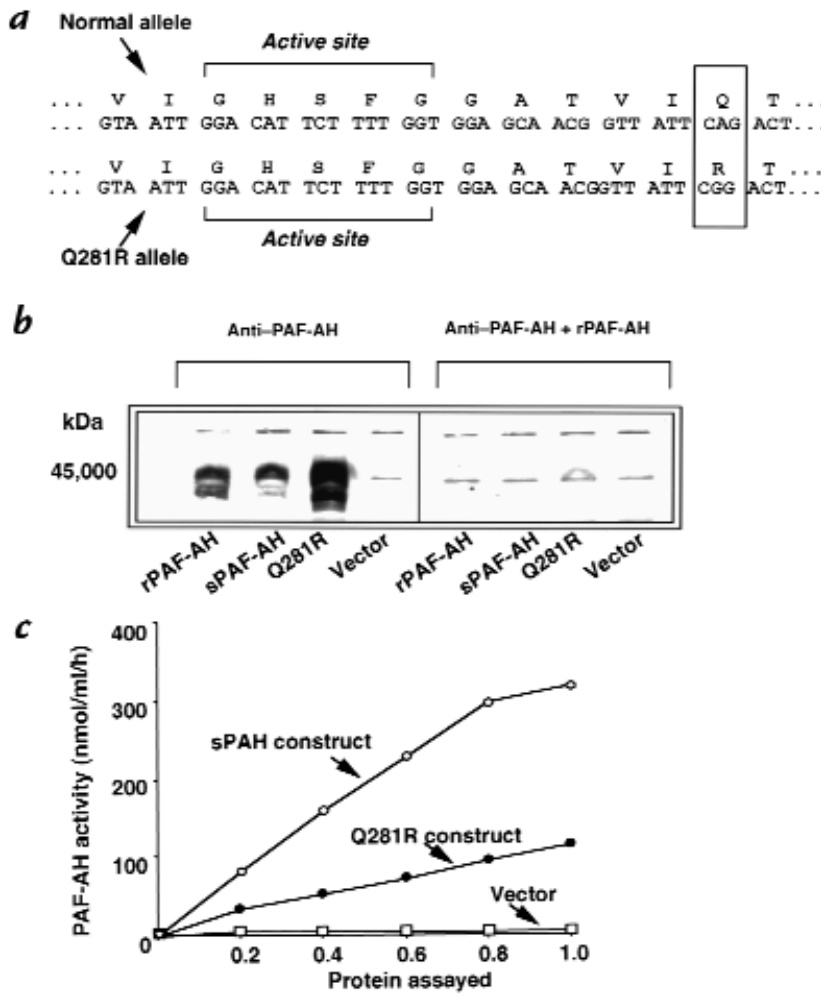

Figure 6

Characterization of an additional mutation (Q281R) that causes partial PAF acetylhydrolase deficiency. (a) The sequence of a mutation found in an asthmatic subject is shown. The Q281R mutation maps near the active site. (b) Immunoblot showing expression of the Q281R mutant in E. coli. (c) PAF acetylhydrolase activity determination of the Q281R mutant protein and its comparison with the wild type and with the vector control.

mutation in only one allele and was therefore included in the heterozygous group. The activity levels of this subject clearly fell outside the range of activity covered by the rest of the asthmatic heterozygous group (see Figure $3 a$ ). The unexpectedly low activity level could have resulted from acquired deficiency $(39,40)$, or conversely, could have been the consequence of additional mutations associated with loss of function. To test the second hypothesis, we amplified all exons using genomic DNA isolated from blood cells and found that the coding sequence of the allele that did not harbor the V279F mutation had a different mutation in exon 9, near the active site (41; Q281R, Figure $6 a)$. This mutation was previously described, by Yamada and Yokota (42), in a Japanese patient with vascular disease. The sequence of all other exons was normal (data not shown). Thus, this asthmatic individual was a compound heterozygote that harbored the V279F mutation in one allele and the Q281R mutation in the other. To determine if the Q281R mutation results in altered enzymatic activity, we expressed the mutant in E. coli and found that PAF acetylhydrolase protein was expressed at levels similar to those of the wild-type control (Figure $6 b$ ). However, the Q281R mutant had a much lower level of activity than did the wild type (22\%; Figure 6c).

In addition to Q281R, we detected two additional alterations in the PAF acetylhydrolase gene in this group of subjects. First, we found that several subjects harbored a deletion in the intron located upstream of exon 12 (Figure 7). The deletion did not correlate with activity levels (data not shown) and probably represents a polymorphism. We also found a previously described polymorphism (43) in exon 7, I198T (Figure 8). This polymorphism results in the transversion of isoleucine 198 to threonine and was present in most samples that harbored the V279F mutation. To test whether this amino acid substitution altered PAF acetylhydrolase activity, we expressed the I198T mutant in E. coli (Figure 8b). The enzymatic activity expressed by the mutant was similar to that of the wild-type control (Figure 8c).

\section{Discussion}

We have investigated the possibility that PAF acetylhydrolase deficiency is a risk factor for asthma in Japanese adults. A commonly occurring mutation, V279F, that results in the partial (in heterozygotes) or complete (in homozygotes) lack of enzymatic activity occurs in Japan, and the presence of this mutation and the total amount of PAF acetylhydrolase activity in plasma were the basis of our screening assays. We found that deficiency of PAF acetylhydrolase constitutes a risk factor for asthma and that completely deficient subjects are more likely to develop severe forms of the disease. In addition, our statistical analysis indicates that a deficiency in the enzymatic activity increases the odds of suffering an asthma attack.

The V279F mutation has not been found in white populations. However, these studies have important implications, not only for Asians but also for populations in which inherited PAF acetylhydrolase deficiency is rare, because acquired deficiency has been described in several syndromes $(44,45)$ and in response to hormonal stimuli $(37,38)$. In addition, the range of activity in whites is broad and includes many individuals with activity levels similar to or lower than those of Japanese subjects who are heterozygous for the V279F mutation.

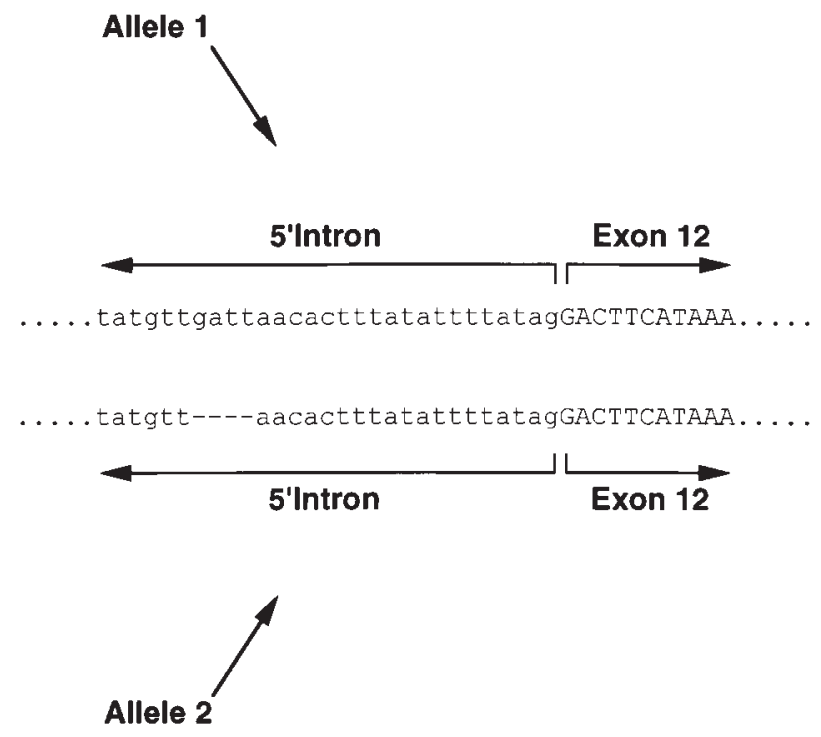

Figure 7

Sequence of a novel polymorphism in the intron that lies $5^{\prime}$ to exon 12 in the PAF acetylhydrolase gene. 
a

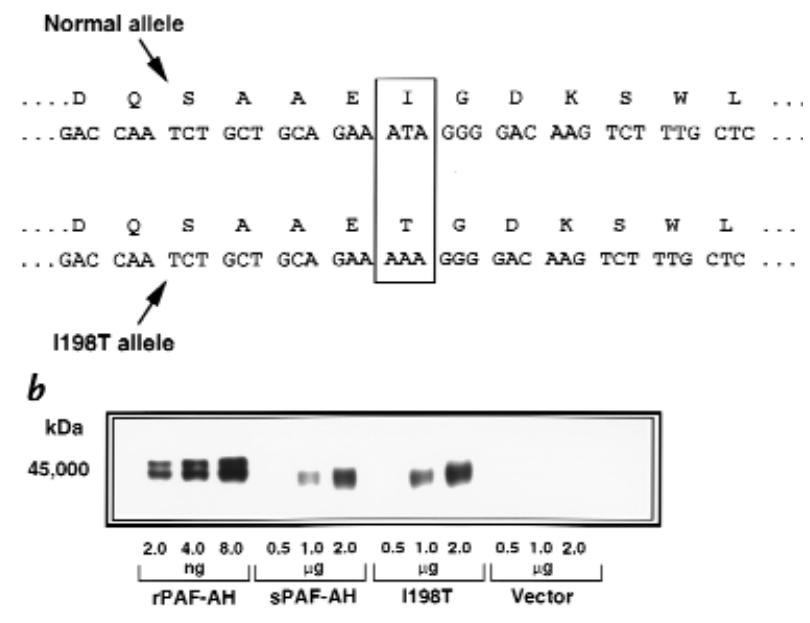

$c$

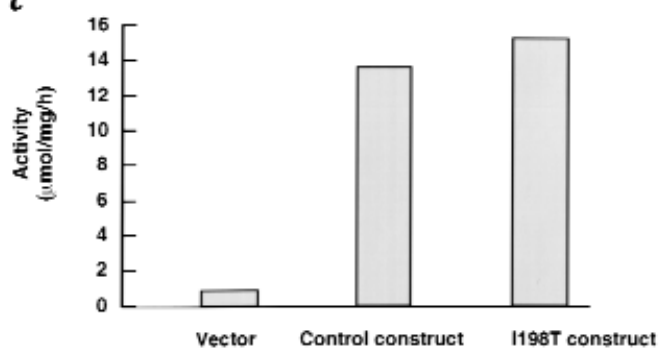

Figure 8

Characterization of a polymorphism (I198T) that has no effect on PAF acetylhydrolase activity. (a) The sequence of a polymorphism found in asthmatic subjects that also harbored the V279F mutation is shown. (b) Immunoblot showing expression of the 1198T mutant in E. coli. (c) PAF acetylhydrolase activity determination of the $1198 \mathrm{~T}$ mutant protein and its comparison to the wild type and to the vector control.

In Japan, asthma in childhood is increasing and is correlated with movement of the population from traditional bare and well-ventilated homes to Westernstyle buildings (46). Supporting a role for PAF acetylhydrolase deficiency in the pathogenesis of asthma is the observation made by Nakamura and Labarthe regarding the incidence of mortality from asthma in Japan and in the US (47). These investigators concluded that mortality rates from asthma were highest among the Japanese, followed by US blacks and, finally, US whites. The largest differences between the two populations (which differed by as much as 12.3 -fold) were observed in children $0-4$ years of age and in subjects aged 65 and older, although mortality rates in patients between 5 and 34 years of age did not differ among Japan, Europe, and the US (48). Although the consistency of diagnosis criteria may have varied somewhat between the US and Japan, the fact that the mortality rates for the elderly in Japan were so much higher than in the US seems unlikely to be explained only by such differences, even if some variation does exist (47). These race-specific mortality rates suggest that genetic variations, such as PAF acetylhydrolase mutations, are substantial modifiers of the disease, at least in the elderly population.
We found no differences in the prevalence of severe, moderate persistent, mild persistent or mild intermittent forms of the disease between asthmatic subjects with heterozygous versus normal phenotypes, which suggests that factors other than PAF acetylhydrolase activity become relevant as predictors of severity when that deficiency is only partial. In contrast, a complete deficiency in activity is likely to result in the accumulation of PAF and other biologically active phospholipids (see below) whose cascade-like effects can increase the severity of the disease.

In addition to PAF, another group of compounds that may mediate inflammation in asthma are the oxidatively fragmented phospholipids that display PAF-like activity $(22,49)$. These compounds accumulate in the blood of cigarette smoke-exposed hamsters as a consequence of oxidative reactions (50). Asthma is a condition in which oxidatively fragmented phospholipids also are likely to be produced; elevated hydrogen peroxide levels and thiobarbituric acid-reactive products were detected in expired breath condensate of asthmatic patients (51) and in asthmatic children (52). In addition, stimulated alveolar macrophages from asthmatics generate more reactive oxygen species than alveolar macrophages from healthy subjects (53). This has two implications. First, active oxygen species and oxidants are inhibitors of PAF acetylhydrolase (54). Second, the generation of these active oxygen species can lead to the formation of proinflammatory, oxidatively fragmented phospholipids, which also are degraded by PAF acetylhydrolase $(55,56)$. A decrease in the activity of this enzyme coupled to increased oxidized phospholipid production could plausibly worsen the outcome of asthma.

We found evidence that several genotypes contribute to PAF acetylhydrolase deficiency and a predisposition to asthma, including a novel compound mutation (V279F/Q281R). Yamada and Yokota (42) reported that the Q281R mutation results in the loss of PAF acetylhydrolase activity and that expression of the Q281R cDNA resulted in the production of proteins without enzymatic activity. However, we found that the activity expressed by this mutant was $22 \%$ of that of the wildtype enzyme. The explanation for the differences between our results and those of Yamada and Yokota is not clear, but it possibly resulted from variations in the solubilization buffers used to extract the protein from the E. coli pellet. Our assessment of the activity expressed by the Q281R mutant is likely to be correct, since the total PAF acetylhydrolase activity in the plasma of the compound heterozygous (V279F/Q281R) patient was $0.44 \mathrm{U} / \mathrm{ml}$. Because the normal plasma activity is $3.56 \mathrm{U} / \mathrm{ml}$ (Figures 1 and 2), the activity expressed by the affected subject was $12 \%$ of the control. The activity was thus likely to be the product of the Q281R allele exclusively, because the V279F allele does not contribute activity (24). Therefore, homozygosity for the Q281R mutation would be expected to generate approximately $24 \%$ of the normal plasma activity, which agrees with the measured activity of the recombinant Q281R mutant (22\% of the wild type).

The heterogeneous nature of asthma has made advances in the development of therapies difficult. A key to finding successful new treatments is identification of 
the molecular alterations that account for differences in the natural causes and severity of the syndrome. The studies reported here indicate that genetic alterations that result in decreased plasma PAF acetylhydrolase activity is one such variable. How PAF acetylhydrolase deficiency interacts with other genetically determined and acquired characteristics remains to be investigated. This information is likely to be useful in understanding the features that influence the course and outcome of the asthmatic syndrome in subjects of other races, in addition to those of Japanese descent.

\section{Acknowledgments}

We thank Makoto Fueki and Norio Kihara (Kihara Hospital, Tokyo, Japan) for providing blood samples for this study. Kathryn E. Smith contributed excellent technical assistance, and Diana Lim provided expertise on figure preparation. We acknowledge the DNA Sequencing and Peptide/Oligo DNA Synthesis Core Facilities at the University of Utah (supported by grant CA-42014 from the National Institutes of Health). This work was supported by an award from the American Lung Association (Asthma Research Center Award) and by grants from the National Institutes of Health (HL-35828) and the Huntsman Cancer Foundation.

1. Lemanske, R.F., Jr. and Busse, W.W. 1997. Asthma. JAMA. 278: $1855-1873$

2. Drazen, J.M. 1998. New directions in asthma drug therapy. Hosp. Pract. 33:25-26.

3. Davies, R.J., et al. 1997. New insights into the understanding of asthma. Chest. 111:2S-10S

4. Rachelefsky, G. 1997. Childhood asthma and allergic rhinitis: the role of leukotrienes. J. Pediatr. 131:348-355.

5. Page, C.P. 1988. The role of platelet-activating factor in asthma. J. Allergy Clin. Immunol. 81:44-152.

6. Nadel, J.A. 1996. Genetics reveals importance of platelet activating factor in asthma and possibly other inflammatory states. J. Clin. Invest. 97:2689-2690.

7. Imaizumi, T.A., et al. 1995. Platelet-activating factor: a mediator for clinicians. J. Intern. Med. 238:5-20.

8. Kurosawa, M., Yamashita, T., and Kurimoto, F. 1994. Increased levels of blood platelet-activating factor in bronchial asthmatic patients with active symptoms. Allergy. 49:60-63.

9. Nakamura, T., et al. 1987. Platelet-activating factor in late asthmatic response. Int. Arch. Allergy Appl. Immunol. 82:57-61.

10. Hsieh, K.H., and Ng, C.K. 1993. Increased plasma platelet-activating factor in children with acute asthmatic attacks and decreased in vivo and in vitro production of platelet-activating factor after immunotherapy. $J$. Allergy Clin. Immunol. 91:650-657.

11. Beer, J.H., Wuthrich, B., and von Felten, A. 1995. Allergen exposure in acute asthma causes the release of platelet-activating factor (PAF) as demonstrated by the desensitization of platelets to PAF. Int. Arch. Allergy Immunol. 106:291-296.

12. Nagase, T., et al. 1997. Airway responsiveness in transgenic mice overexpressing platelet- activating factor receptor. Roles of thromboxanes and leukotrienes. Am. J. Respir. Crit. Care Med. 156:1621-1627.

13. Shirasaki, H., et al. 1994. Expression of platelet-activating factor receptor mRNA in human and guinea pig lung. Am. J. Respir. Cell Mol. Biol. 10:533-537.

14. Kuitert, L., and Barnes, N.C. 1995. PAF and asthma-time for an appraisal? Clin. Exp. Allergy. 25:1159-1162.

15. Zimmerman, G.A., McIntyre, T.M., Prescott, S.M., and Stafforini, D.M. 1996. Platelet-activating factor: antagonists, terminators, molecular mimics, and microbial opportunism. J. Intern. Med. 239:463-466.

16. Evans, D.J., Barnes, P.J., Cluzel, M., and O’Connor, B.J. 1997. Effects of a potent platelet-activating factor antagonist, SR27417A, on allergeninduced asthmatic responses. Am. J. Respir. Crit. Care Med. 156:11-16.

17. Tamura, G., et al. 1996. Effect of a potent platelet-activating factor antagonist, WEB-2086, on asthma. A multicenter, double-blind placebo-controlled study in Japan. Adv. Exp. Med. Biol. 416:371-380.

18. Hozawa, S., Haruta, Y., Ishioka, S., and Yamakido, M. 1995. Effects of a PAF antagonist, Y-24180, on bronchial hyperresponsiveness in patients with asthma. Am. J. Respir. Crit. Care Med. 152:1198-1202.

19. Arima, M., Yukawa, T., and Makino, S. 1995. Effect of YM264 on the airway hyperresponsiveness and the late asthmatic response in a guinea pig model of asthma. Chest. 108:529-534.

20. Kagoshima, M., et al. 1997. Effects of Y-24180, a long-acting and potent antagonist to platelet-activating factor, on immediate asthmatic response in guinea pigs. Pharmacology. 54:1-7.

21. Bazan, N.G. 1995. Inflammation. A signal terminator. Nature. 374:501-502.

22. Stafforini, D.M., McIntyre, T.M., Zimmerman, G.A., and Prescott, S.M. 1997. Platelet-activating factor acetylhydrolases. J. Biol. Chem. 272:17895-17898.

23. Miwa, M., et al. 1988. Characterization of serum platelet-activating factor (PAF) acetylhydrolase. Correlation between deficiency of serum PAF acetylhydrolase and respiratory symptoms in asthmatic children. J. Clin. Invest. 82:1983-1991.

24. Stafforini, D.M., et al. 1996. Platelet-activating factor acetylhydrolase deficiency. A missense mutation near the active site of an anti-inflammatory phospholipase. J. Clin. Invest. 97:2784-2791.

25. Grossman, J. 1997. One airway, one disease. Chest. 111:11S-16S

26. 1997. The genetics of asthma. Am. J. Respir. Crit. Care Med. 156:569-S162.

27. In, K.H., et al. 1997. Naturally occurring mutations in the human 5lipoxygenase gene promoter that modify transcription factor binding and reporter gene transcription. J. Clin. Invest. 99:1130-1137.

28. National Asthma Education and Prevention Program. 1997. Expert panel report. II. Guidelines for the diagnosis and management of asthma. NIH publication 97-4051A. National Institutes of Health. Bethesda, MD.

29. Stafforini, D.M., McIntyre, T.M., and Prescott, S.M. 1990. Platelet-activating factor acetylhydrolase from human plasma. Methods Enzymol. 187:344-357.

30. Tjoelker, L.W., et al. 1995. Plasma platelet-activating factor acetylhydrolase is a secreted phospholipase A2 with a catalytic triad. J. Biol. Chem. 270:25481-25487.

31. McCullaugh, P. Regression models of ordinal data. 1980. J. Royal Stat. Soc. 42:109-142

32. Tsodikov, A., Hasenclever, D., and Loeffler, M. 1998. Regression with bounded outcome score: evaluation of power by bootsstrap and simulation in a chronic myelogenous leukaemia clinical trial. Stat. Med. 17:1909-1922.

33. Tsukioka, K., Matsuzaki, M., Nakamata, M., and Kayahara, H. 1993. Increased plasma levels of platelet-activating factor (PAF) and low serum PAF acetylhydrolase (PAFAH) activity in adult patients with bronchial asthma. Arerugi. 42:167-171.

34. Tsukioka, K., Matsuzaki, M., Nakamata, M., Kayahara, H., and Nakagawa, T. 1996. Increased plasma level of platelet-activating factor (PAF) and decreased serum PAF acetylhydrolase (PAFAH) activity in adults with bronchial asthma. J. Investig. Allergol. Clin. Immunol. 6:22-29.

35. Miyaura, S., Eguchi, H., and Johnston, J.M. 1992. Effect of a cigarette smoke extract on the metabolism of the proinflammatory autacoid, platelet-activating factor. Circ. Res. 70:341-347.

36. Imaizumi, T., et al. 1990. Activity of platelet-activating factor (PAF) acetylhydrolase in plasma from healthy habitual cigarette smokers. Heart Vessels. 5:81-86.

37. Yasuda, K., and Johnston, J.M. 1992. The hormonal regulation of plateletactivating factor-acetylhydrolase in the rat. Endocrinology. 130:708-716.

38. Miyaura, S., Maki, N., Byrd, W., and Johnston, J.M. 1991. The hormonal regulation of platelet-activating factor acetylhydrolase activity in plasma. Lipids. 26:1015-1020.

39. Maki, N., Hoffman, D.R., and Johnston, J.M. 1988. Platelet-activating factor acetylhydrolase activity in maternal, fetal, and newborn rabbit plasma during pregnancy and lactation. Proc. Natl. Acad. Sci. USA. 85:728-732.

40. Yasuda, K., Takashima, M., and Sawaragi, I. 1995. Influence of a cigarette smoke extract on the hormonal regulation of platelet-activating factor acetylhydrolase in rats. Biol. Reprod. 53:244-252.

41. Tjoelker, L.W., et al. 1995. Anti-inflammatory properties of a plateletactivating factor acetylhydrolase. Nature. 374:549-553.

42. Yamada, Y., and Yokota, M. 1997. Loss of activity of plasma platelet-activating factor acetylhydrolase due to a novel Gln281 $\rightarrow$ Arg mutation. Biochem. Biophys. Res. Commun. 236:772-775.

43. Bell, R., et al. 1997. Systematic screening of the LDL-PLA2 gene for polymorphic variants and case-control analysis in schizophrenia. Biochem. Biophys. Res. Commun. 241:630-635.

44. Tetta, C., et al. 1990. Release of platelet-activating factor in systemic lupus erythematosus. Int. Arch. Allergy Appl. Immunol. 91:244-256.

45. Graham, R.M., et al. 1994. Platelet and plasma platelet-activating factor in sepsis and myocardial infarction. J. Lipid Mediat. Cell Signal. 9:167-182.

46. Cookson, W.O., and Moffatt, M.F. 1997. Asthma: an epidemic in the absence of infection? Science. 275:41-42.

47. Nakamura, Y., and Labarthe, D.R. 1994. Secular trends in mortality from asthma in Japan, 1979-1988: comparison with the United States. Int. J. Epidemiol. 23:143-147.

48. National Heart, Lung and Blood Institution and the World Health Organization. 1995. Global initiative for asthema. NIH publication 95-3659. 
National Institutes of Health. Bethesda, MD

49. Zimmerman, G.A., Prescott, S.M., and McIntyre, T.M. 1995. Oxidatively fragmented phospholipids as inflammatory mediators: the dark side of polyunsaturated lipids. J. Nutr. 125:1661S-1665S

50. Lehr, H.A., et al. 1997. Vitamin C blocks inflammatory platelet-activating factor mimetics created by cigarette smoking. J. Clin. Invest. 99:2358-2364.

51. Antczak, A., et al. 1997. Increased hydrogen peroxide and thiobarbituric acid-reactive products in expired breath condensate of asthmatic patients. Eur. Respir. J. 10:1235-1241.

52. Jöbsis, Q., Raatgeep, H.C., Hermans, P.W., and de Jongste, J.C. 1997. Hydrogen peroxide in exhaled air is increased in stable asthmatic children. Eur. Respir. J. 10:519-521.
53. Cluzel, M., et al. 1987. Enhanced alveolar cell luminol-dependent chemiluminescence in asthma. J. Allergy Clin. Immunol. 80:195-201.

54. Ambrosio, G., et al. 1994. Oxygen radicals inhibit human plasma acetylhydrolase, the enzyme that catabolizes platelet-activating factor. J. Clin. Invest. 93:2408-2416.

55. Stremler, K.E., et al. 1989. An oxidized derivative of phosphatidylcholine is a substrate for the platelet-activating factor acetylhydrolase from human plasma. J. Biol. Chem. 264:5331-5334.

56. Stremler, K.E., Stafforini, D.M., Prescott, S.M., and McIntyre, T.M. 1991. Human plasma platelet-activating factor acetylhydrolase. Oxidatively fragmented phospholipids as substrates. J. Biol. Chem. 266:11095-11103. 